\title{
Fatigue failure load of immature anterior teeth: influence of different fiber post-core systems
}

\author{
Márk Fráter $^{1} \cdot$ Tekla Sáry $^{1} \cdot$ Viktória Néma $^{1} \cdot$ Gábor Braunitzer $^{2} \cdot$ Pekka Vallittu $^{3,4} \cdot$ Lippo Lassila $^{3} \cdot$ Sufyan Garoushi ${ }^{3}$
}

Received: 29 January 2020 / Accepted: 17 April 2020

(c) The Society of The Nippon Dental University 2020

\begin{abstract}
The aim was to explore the fracture-behavior, survival and marginal-microgaps within the root-canal of immature anterior teeth restored with different fiber-reinforced post-core composites (FRCs). 180 bovine-incisors were randomly divided into 6 groups $(n=30)$. One group served as control (group 6). The rest of the teeth were prepared to an internal diameter of $1.6 \mathrm{~mm}$ and the apex was sectioned. After application of an MTA-plug, teeth were restored with FRC materials: Group 1: Bioblock technique with short fiber-reinforced composite (SFRC); Group 2: Bioblock technique with flowable SFRC; Group 3: Individually-made FRC post; Group 4: Conventional FRC post; Group 5: dual-cure core build-up composite. After restorations were completed, teeth ( $n=5 /$ group) were sectioned and then stained. Specimens were viewed under a stereo microscope and the percentage of microgaps within the root-canal was calculated. Fatigue-survival was measured using a cyclic-loading testing machine in the rest of the specimens. Flowable SFRC application in the root-canal (Group 2) did not differ from intact-teeth regarding fatigue-survival $(p>0.05)$. The rest of the groups produced significantly lower survival $(p<0.05)$ compared to intact-teeth. Post/core restorations made from conventional FRC post (Group 4) exhibited a high number of microgaps (38.3\%) at the examined interphase in the root-canal. The restoration of immature incisors with the use of flowable SFRC as post-core material displayed promising performance in a matter of fatigue-resistance and survival.
\end{abstract}

Keywords Immature teeth $\cdot$ Short fiber-reinforced composite $\cdot$ Fatigue survival $\cdot$ Bioblock technique $\cdot$ Fracture behavior

\section{Introduction}

Traumatic dental injuries to developing teeth are common in patients between the ages of 8 and 12 years [1] and mostly affect the maxillary central incisors [2]. These injuries often lead to pulpal necrosis with a subsequent cessation of root formation [3]. The result is the formation of an immature tooth with divergent, thin dentinal walls and an open apex. Subsequently, these teeth are highly susceptible to fracture, especially in the cervical area [4]. As retaining these teeth

Márk Fráter

sufgar@utu.fi

1 Department of Operative and Esthetic Dentistry, Faculty of Dentistry, University of Szeged, Szeged, Hungary

2 dicomLAB Dental Ltd, Szeged, Hungary

3 Department of Biomaterials Science and Turku Clinical Biomaterials Center-TCBC Institute of Dentistry, University of Turku, Itäinen Pitkäkatu 4 B, 20520 Turku, Finland

4 City of Turku, Welfare Division, Turku, Finland during the craniofacial growth of the patients is critical, the primary treatment goal is to reinforce them. To restore the weakened dental structure, the open apex must be managed first. The traditional approach is apexification using longterm calcium hydroxide therapy, but it has been largely replaced by the application of mineral trioxide aggregate (MTA) apical plugs [5]. The latter procedure, known as oneshot apexification, has several advantages, such as a shorter treatment time and the establishment of a proper apical seal [6, 7]. Albeit the MTA barrier can easily manage and close the open apex, it cannot adequately strengthen the weak and thin dentinal walls. Application of a fiber-reinforced composite (FRC) post after the MTA plug was suggested to reinforce these traumatized teeth [8]. As shown by many, immature anterior teeth are highly susceptible to cervical root fracture, even from normal masticatory forces [3, 9]. Elnaghy et al. emphasized that the high frequency of cervical root fractures in immature teeth is predominantly due to fatigue crack growth over time, not secondary to trauma [10]. Thus, the fate of immature teeth after MTA apexification is determined partly by the integrity of the remaining 
tooth structure and partly by the permanent restoration to keep it in function [11]. However, traditional post insertion requires the usage of post drills, which hold the possibility of further weakening the root by removing dentine. In additon, as posts are luted with dual-cure resin cements to the root canal walls and mostly not closely adapted to them, their reinforcing effect is questionable. The application of short fiber-reinforced composites (SFRC) inside the root canal has been recommended by several authors [12-14]. In the Bioblock technique, a packable SFRC (everX Posterior, GC Europe, Leuven, Belgium) is luted directly to the canal walls in a bulk manner [14]. By this, not only the unique root canal is filled individually, but also the need for a luting cement is eliminated, possibly providing one step forward to a monoblock FRC restoration. In 2019, the flowable version of SFRC has been launched with the promise of easy adaptability in limited spaces (e.g. root canals). So far, many studies have been carried out on the material itself [15-17] but none has used it to restore and reinforce root canal treated teeth.

The purpose of the present in vitro investigation was to assess the fatigue resistance and failure mode of simulated immature teeth after one-shot apexification restored with different FRC materials. In addition, the curing performance at different depths and adaptation of materials within the root canal for each restorative option were investigated.

\section{Materials and methods}

Four hundred upper bovine-incisors were collected and stored in $0.5 \%$ chloramine-T. The largest facial-lingual and mesio-distal dimensions of the coronal part and also the hight of the coronal portion from the cementoenamel junction (CEJ) were measured. On the basis of the measurements, only teeth with a maximum deviation of $10 \%$ from the determined mean were included in this study. Also the facial-lingual and mesio-distal dimensions of the root part were measured in order to standardize the size and thickness of the roots. Teeth with a variation of more than $10 \%$ from criterion dimensions regarding the root $(4.5 \mathrm{~mm}$ for mesio-distal and $6 \mathrm{~mm}$ for facial-lingual) were excluded. Finally, a sum of one hundred eighty teeth was included into the study. Teeth were randomly distributed over 6 study groups $(n=30)$. One group was left intact to serve as a control (Group 6).

The rest of the teeth (Group 1-5) were sectioned to obtain a length of $12 \mathrm{~mm}$ below the CEJ using a slow-speed, watercooled diamond disc. Furthermore, after sectioning of the apical part, all teeth were examined under magnification for root fractures and the ones with a fracture or visible crack or any external resorption were excluded from the study and replaced with one with adequate parameters.

\section{Specimen preparation and restorative procedures}

Coronal access was made by using a round-end parallel diamond (881.31.014 FG-Brasseler USA Dental, Savannah, GA, USA) and an Endo Z bur (Dentsply Maillefer, Tulsa, OK, USA) in a high-speed handpiece. Next, the root canal was enlarged by Gates Glidden burs No. 1-6 with copious water cooling until an ISO size \#140 could be passively extended through the apex. Each canal was then prepared with a GC Fiber Post drill size 1.6 (GC Europe, Leuven, Belgium) to simulate an immature tooth with thin walls. Each tooth was irrigated with $5 \mathrm{~mL} 5 \% \mathrm{NaOCl}$ and $5 \mathrm{~mL} 17 \%$ ethylenediaminetetraacetic acid (EDTA), followed by $5 \mathrm{~mL}$ of sterile water as the final rinse. A 4-mm apical plug of grey Pro-Root MTA (Dentsply Tulsa Dental, Tulsa, OK, USA) was placed in each tooth with a MAP System (Dentsply Maillefer, Tulsa, OK, USA). The teeth were stored at $37{ }^{\circ} \mathrm{C}$ and $100 \%$ humidity for $48 \mathrm{~h}$. After complete setting of the MTA was confirmed with an endodontic explorer and before restorative procedures, the radicular dentine was refreshed with a No. 4 Gates Glidden bur and flushed with chlorhexidine and saline. The enamel borders of the coronal cavity were acid-etched selectively with $37 \%$ phosphoric acid (Ultra-Etch, Ultradent Products, South Jordan, UT) for $15 \mathrm{~s}$ and rinsed with water. The root canals were dried with paper points. For bonding, a dual-cure one-step self-etch adhesive system (G-Premio Bond and DCA, GC Europe, Leuven, Belgium) was used, according to the manufacturer's instructions using a microbrush-X disposable applicator (Pentron Clinical Technologies, LLC, USA). Excess adhesive was removed by suction drying (Evacuation Tip-Starryshine, Anaheim, CA, USA) within $0.5 \mathrm{~cm}$ from the occlusal cavity (without contact). Excess adhesive resin at the bottom of the canal was removed with a paper point. The adhesive was light cured for $60 \mathrm{~s}$ using an Optilux 501 quartztungsten-halogen light-curing unit (Kerr Corp., Orange, CA, USA). The average power density of the light source, measured with a digital radiometer (Jetlite light tester; J. Morita USA Inc. Irvine, CA, USA) prior to the bonding procedure, was $840 \pm 26.8 \mathrm{~mW} / \mathrm{cm}^{2}$. The light source was placed as close as possible to the coronal cavity without achieving direct contact with it.

The teeth in all groups were then treated as follows (Fig. 1.):

Group 1: The teeth were reconstructed with the Bioblock technique described by Fráter et al. [14] building a direct layered post and core from packable SFRC (everX Posterior). An approx. $4 \mathrm{~mm}$ thick increment of SFRC was packed to the apical portion of the post space 
Fig. 1 Shematic figure representing the test groups (Group 1-5). Gr. 1: Bioblock technique with packable SFRC; Gr. 2: Bioblock technique with flowable SFRC; Gr. 3: Individuallymade unidirectional FRC post; Gr. 4: Conventional unidirectional FRC post; Gr. 5: Dualcure core build-up material

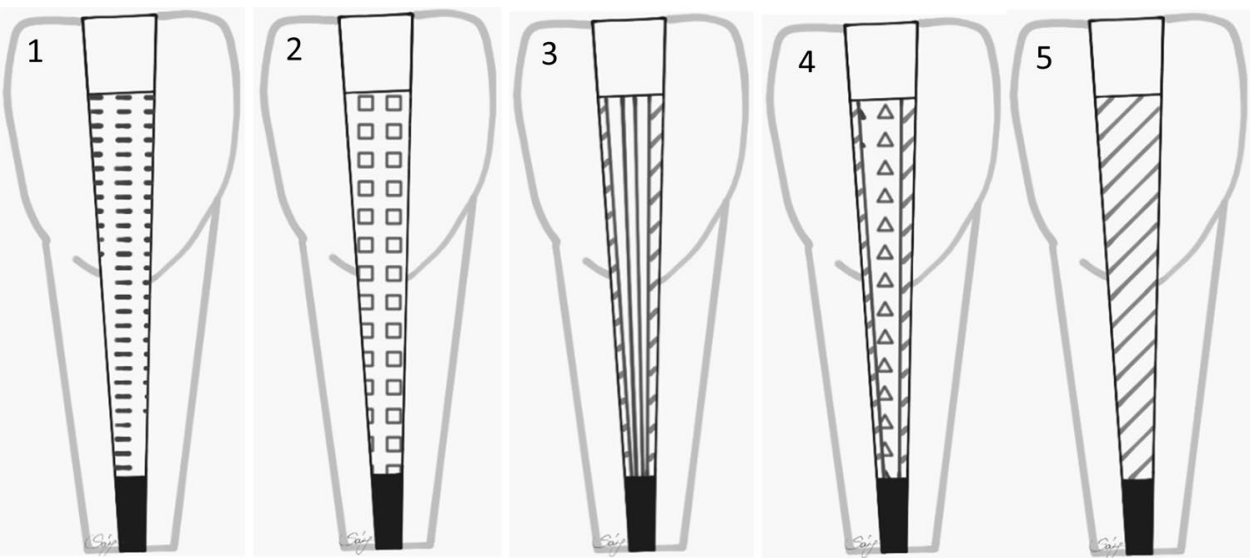

using a microbrush-X disposable applicator (Pentron Clinical Technologies, LLC, USA). A light transmitting FRC post (1.4 mm GC Fiber post, GC Europe, Leuven, Belgium) was inserted into the post space to aid the transmission of the light to the apically positioned layers. The 'light transmitting' post was withdrawn with $0.5-1 \mathrm{~mm}$ from the surface of the uncured SFRC layer not to have direct contact with it. After each layer, $80 \mathrm{~s}$ of light curing through the fiber post was carried out. After incrementally filling the root canal to the level of the CEJ with repeating the previously described procedure, SFRC was layered in the coronal cavity until $1 \mathrm{~mm}$ below the margin of the occlusal cavity in a concave shape. Each coronally placed increment was light cured from the occlusal surface for $40 \mathrm{~s}$. The last $2 \mathrm{~mm}$ thick surface layer was composite material (G-aenial Anterior JE, GC Europe, Leuven, Belgium) covering the SFRC.

Group 2: The teeth were reconstructed with flowable SFRC (everX Flow, GC Europe, Leuven, Belgium) as described in Group 1.

Group 3: The teeth received a $1.5 \mathrm{~mm}$ diameter individually-made unidirectional FRC post (everStick Post, GC Europe, Leuven, Belgium). Luting of the posts and the core build-up was performed with a dual-cure resin composite core material (Gradia Core, GC Europe, Leuven, Belgium). Gradia Core was applied using its own automix cartridge with an 'elongation tip' for direct root canal application. After the insertion of the post, the composite core material was polymerized from the top of the post with an Optilux 501. quartz-tungsten-halogen lightcuring unit for $60 \mathrm{~s}$ from each side (a total of $240 \mathrm{~s} /$ tooth). The last $2 \mathrm{~mm}$ thick surface layer was composite material as in Group 1.

Group 4: The teeth received a $1.6 \mathrm{~mm}$ diameter conventional unidirectional FRC post (GC Fiber Post, GC Europe, Leuven, Belgium). The conventional translucent FRC posts were tried in and cut to a length $2 \mathrm{~mm}$ below the level of the occlusal cavity margins with a watercooled diamond disc (Isomet 2000; Buehler Ltd., Lake Bluff, IL, USA) and cleaned with alcohol after try in. The posts received silanization of the surface (Ceramic Primer II, GC Europe, Leuven, Belgium) following the manufacturer's recommendation. After silanization, the post surface was bonded with the same bonding agent used for the cavity. Luting of the posts and the core build-up was performed with a dual-cure resin composite core material (Gradia Core) as in Group 3. The last $2 \mathrm{~mm}$ thick surface layer was composite material as in Group 1.

Group 5: The teeth were reconstructed without any FRC material, with a dual-cure resin composite core material (Gradia Core). Gradia Core was applied using its own automix cartridge with an 'elongation tip' for direct root canal application. Both the root canal and the coronal cavity was filled up with the core material. The light curing was the same as in Group 3. The last $2 \mathrm{~mm}$ thick surface layer was composite material as in Group 1.

Finally, for all restored teeth, glycerine gel (DeOx Gel, Ultradent Products Inc., Orange, CA, USA) was applied and final polymerization from each side for $40 \mathrm{~s}$ was performed. The light source was placed as close as possible to the coronal cavity without achieving direct contact with it. The restorations were finished with a fine granular diamond burr (FG 7406-018, Jet Diamonds, USA and FG 249-F012, Horico, Germany) and aluminum oxide polishers (OneGloss PS Midi, Shofu Dental GmbH, Ratingen, Germany). After the restorative procedures mechanical testing was carried out on 25 teeth from each group $(n=150)$ and 5 teeth from each restored group $(n=25)$ underwent sectioning, microleakage and microhardness testing.

\section{Embedding and mechanical testing of specimen}

The restored specimens were stored in distilled water at $37^{\circ} \mathrm{C}$ for a week. To simulate the periodontal ligament, the 
root surface of each tooth was coated with a layer of liquid latex separating material (Rubber-Sep, Kerr, Orange, CA) prior to embedding. Specimens were embedded in methacrylate resin (Technovit 4004, Heraeus-Kulzer) at $2 \mathrm{~mm}$ from the cementoenamel junction (CEJ) to simulate the bone level.

For mechanical testing, the restored specimens were submitted to a modified accelerated fatigue-testing protocol by a hydraulic testing machine (Instron ElektroPlus E3000, Norwood, MA, USA) at an angle of 135 degrees to the long axis of each tooth. Cyclic isometric loading was applied on the palatal surface of the coronal part of the tooth using a round-shaped metallic tip (Fig. 2). A cyclic load was applied at a frequency of $5 \mathrm{~Hz}$, starting with gradually increasing static loading till $100 \mathrm{~N}$ in $5 \mathrm{~s}$, followed by cyclic loading in stages of $100 \mathrm{~N}, 200 \mathrm{~N}, 300 \mathrm{~N}, 400 \mathrm{~N}, 500 \mathrm{~N}$ and $600 \mathrm{~N}$ at 5000 cycles each. The specimens were loaded until fracture occurred or a total of 30,000 cycles for the whole procedure. For the survival analyses, the amount of cycles at which the specimen failed were recorded. The failed specimens were visually examined for the type and location of failure, as well as the direction of failure. According to Scotti and co-workers, a distinction was made between restorable or non-restorable fractures under an optical microscope with a two-examiner agreement. A restorable fracture is above

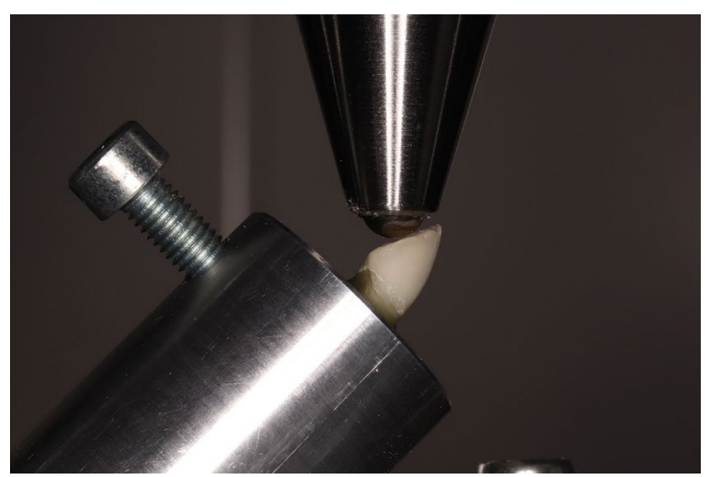

Fig. 2 Embedded specimen loaded at 135 degree on the palatal surface the CEJ, meaning that in case of fracture, the tooth can be restored, while a non-restorable fracture extends below the CEJ and the tooth is likely to be extracted [18]. The data were statistically analyzed with SPSS software (SPSS ver. 23, IBM Corp., Somers, NJ, USA).

\section{Gap visualization test}

Five groups, each consisting of 5 endodontically treated and restored teeth, were investigated in the gap visualization test. The teeth were restored in the same way as mentioned earlier (Group 1-5). Teeth were sectioned mid-sagitally in the mesio-distal plane using a ceramic cutting disc operating at a speed of $100 \mathrm{rpm}$ (Struers, Glasgow, Scotland) under water cooling. In each group, one of the sectioned restorations that contain the post was further ground and polish using \#4000-grit silicon carbide papers at $300 \mathrm{rpm}$ under water cooling using an automatic grinding machine (Rotopol-1; Struers, Copenhagen, Denmark). Then, sectioned teeth were painted with a permanent marker, and polish gently for a few seconds. The dye penetration along post/core margins of each section was evaluated independently using a stereomicroscope (Heerbrugg M3Z, Heerbrugg, Switzerland) at a magnification of $6.5 \times$ and the extent of dye penetration was recorded in $\mathrm{mm}$ as a percentage of the total margin length (Fig. 3).

\section{Microhardness test}

Microhardness of luting composite and SFRCs inside the canal was measured using a Struers Duramin hardness microscope (Struers, Copenhagen, Denmark) with a 40 objective lens and a load of $1.96 \mathrm{~N}$ applied for $10 \mathrm{~s}$. Each sectioned restoration was subjected to 5 indentations on the top, middle and bottom of the canal. The diagonal length impressions were measured and Vickers values were converted into microhardness values by the machine. Microhardness was obtained using the following equation:
Fig. 3 Pictures of sectioned specimens (Groups 4 and 5) showing microgaps at resin composite-root canal interface

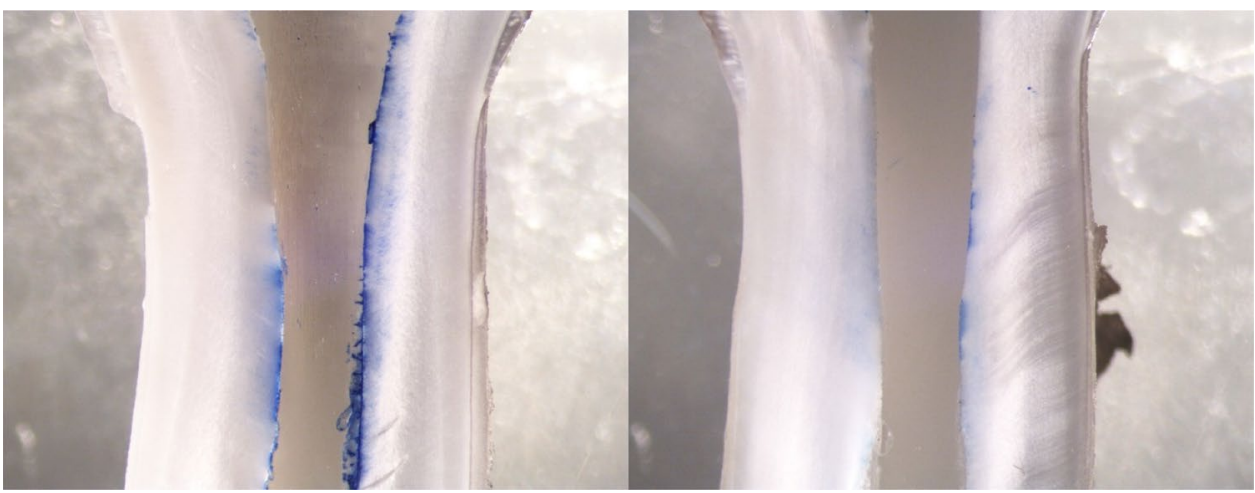




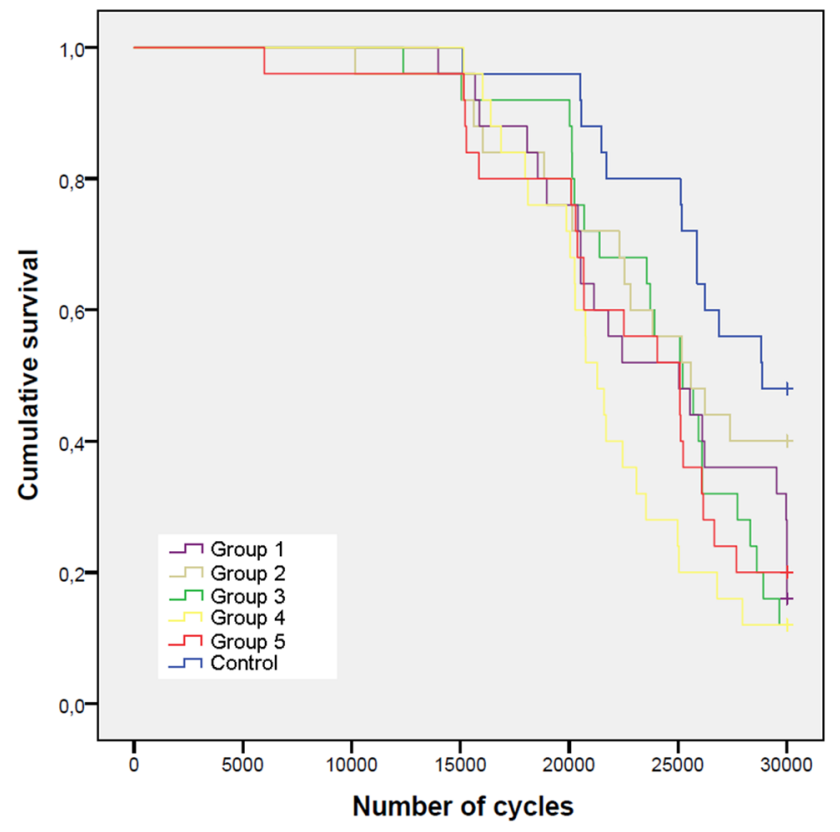

Fig. 4 Fatigue resistance survival curves (Kaplan-Meier survival estimator) for all six groups. Bioblock technique with packable SFRC (Group 1); Bioblock technique with flowable SFRC (Group 2); individually-made FRC post (Group 3); conventional FRC post (Group 4); dual-cure core build-up material (Group 5)

$H=\frac{1854.4 \times P}{d^{2}}$

where $H$ is Vickers hardness in $\mathrm{kg} / \mathrm{mm}^{2}, P$ is the load in grams and $d$ is the length of the diagonals in $\mu \mathrm{m}$.
Microhardness was used as indicative parameter for degree of cure [19].

\section{Results}

The Kaplan-Meier survival curves are displayed in Fig. 4. Table 1. shows the $p$ values for group-wise comparisons. The survival rate of Group 2 did not differ significantly from the intact teeth (control group). The rest of the groups had significantly lower survival rates compared to the control group.

All restored groups showed exclusively irreparable fractures, whereas the control group had some that were reparable, but most fractures were irreparable (Table 2).

The mean values and standard deviations of microgap percentage at post/core-root canal interface of the five restored groups are presented in Fig. 5. According to our findings, the Bioblock technique (Group 1) had low percentage of microgaps $(19,7 \%)$ compared to the other groups, whereas Group 4 exhibited a remarkably high number of microgaps $(38,3 \%)$ at the examined interphase in the root canal.

The surface microhardness ( $\mathrm{VH}$ ) of the luting composite and SFRCs decreased gradually within a limited range with increasing depth (Fig. 6). The data showed no difference in $\mathrm{VH}$ values between the tested dual-core luting composite and SFRCs at top and middle parts of the canal. However, at apical part, packable SFRC (Group 1) presented the most drastic decrease along with the $\mathrm{VH}$ values.

Table 1 p values of pairwise log-rank post hoc comparisons (Kaplan-Meier survival estimator followed by log-rank test for cycles until failure or the end of the fatigue loading among all 6 groups)

\begin{tabular}{|c|c|c|c|c|c|c|c|c|c|c|c|c|}
\hline \multirow[t]{2}{*}{ Groups } & \multicolumn{2}{|l|}{ Control } & \multicolumn{2}{|l|}{ Group 3} & \multicolumn{2}{|l|}{ Group 2} & \multicolumn{2}{|l|}{ Group 1} & \multicolumn{2}{|l|}{ Group 4} & \multicolumn{2}{|l|}{ Group 5} \\
\hline & Chi square & Sig. & Chi square & Sig. & Chi square & Sig. & Chi square & Sig. & Chi square & Sig. & Chi square & Sig. \\
\hline $\begin{array}{c}\text { Control } \\
\text { (intact } \\
\text { teeth) }\end{array}$ & & & 6.208 & 0.013 & 1.722 & .189 & 5.551 & 0.018 & 13.801 & 0.000 & 7.083 & 0.008 \\
\hline Group 1 & 5.551 & 0.018 & 0.000 & 1.000 & 0.793 & 0.373 & & & 1.434 & 0.231 & 0.143 & 0.705 \\
\hline Group 2 & 1.722 & 0.189 & 0.355 & 0.551 & & & 0.793 & 0.373 & 3.401 & 0.065 & 1.003 & 0.316 \\
\hline Group 3 & 6.208 & 0.013 & & & 0.355 & .551 & 0.000 & 1.000 & 3.467 & 0.063 & .254 & 0.614 \\
\hline Group 4 & 13.801 & 0.000 & 3.467 & 0.063 & 3.401 & 0.065 & 1.434 & 0.231 & & & 1.027 & 0.311 \\
\hline Group 5 & 7.083 & 0.008 & 0.254 & 0.614 & 1.003 & 0.316 & 0.143 & 0.705 & 1.027 & 0.311 & & \\
\hline
\end{tabular}

Table 2 The distribution of fracture pattern among the study groups $(n=25)$

\begin{tabular}{lcccccc}
\hline Fracture pattern & Group 1 & Group 2 & Group 3 & Group 4 & Group 5 & Intact teeth \\
\hline Restorable & 0 & 0 & 0 & 0 & 0 & 2 \\
Non restorable & 18 & 15 & 22 & 22 & 20 & 11 \\
Fractured teeth & 18 & 15 & 22 & 22 & 20 & 13 \\
Non fractured teeth & 7 & 10 & 3 & 3 & 5 & 12 \\
\hline
\end{tabular}


Fig. 5 Mean percentage of microgaps observed in different groups from total post/coretooth interface length after staining. Vertical lines represent standard deviation
Fig. 6 Microhardness (VH) mean values for resin composites at the top (coronal), middle and bottom (apical) part of the root canal. Arrow above the column indicate $\mathrm{VH}$ of this group dropped below $80 \%$ of the coronal part value. Vertical lines represent standard deviation
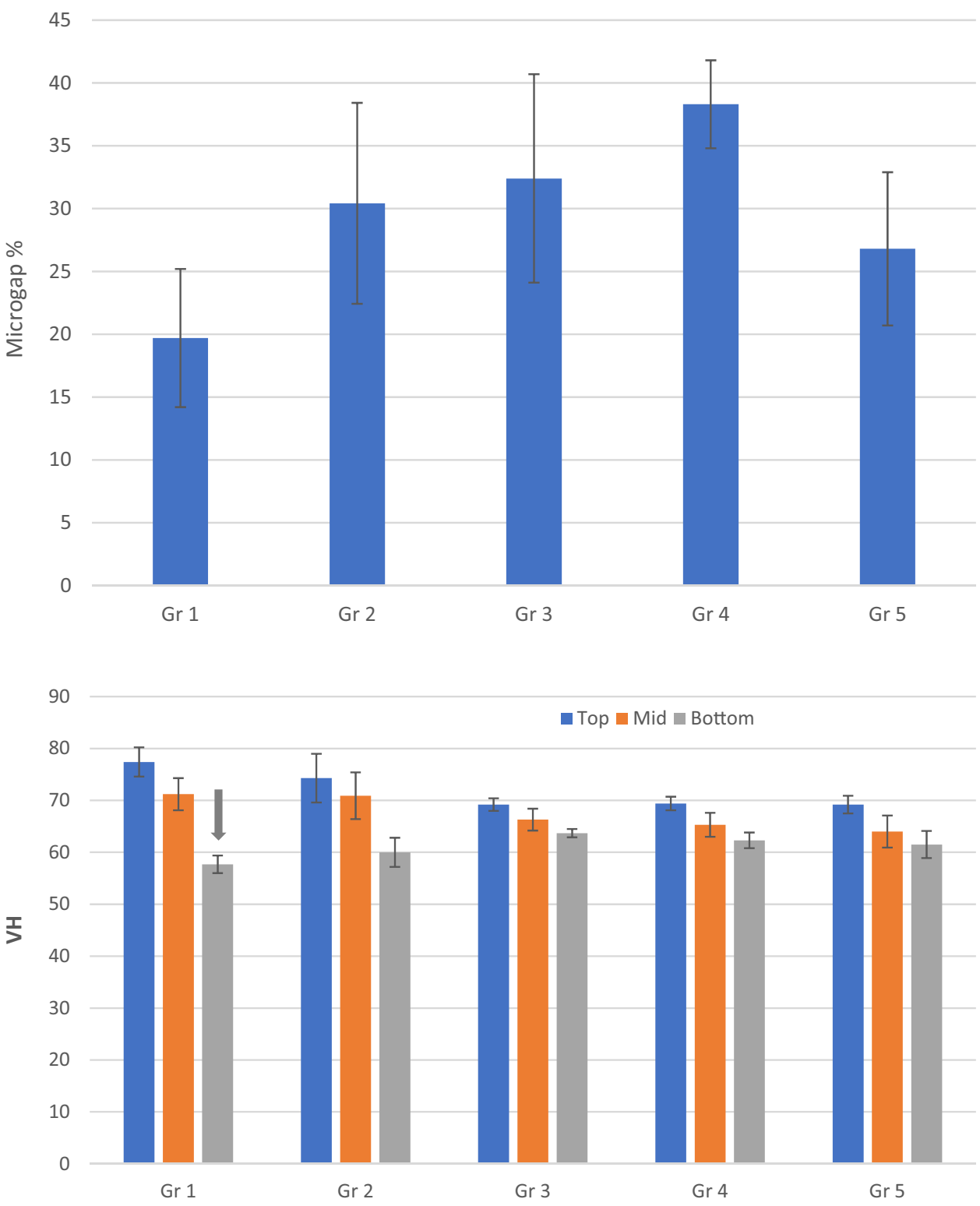

\section{Discussion}

Nowadays the concept of intraradicular reinforcement of immature teeth after one-shot apexification has got increased attention [11]. In our study, various fiber-reinforced composite materials were used with the aim of reinforcing immature teeth. Bovine teeth were used, as extracted human teeth are not readily available and their anatomical variability is quite high. Bovine teeth are normally used when anterior restorations need to be modeled in higher numbers [20,21], as bovine dentine is considered to be similar to human dentine in composition and geometric root configuration [22, 23]. In this study, extreme care was taken to standardize the size of the utilized teeth in all dimensions. In addition, cyclic loading was used instead of static load-to-fracture testing. Cycling loading corresponds better to the clinical situation, as it generates repetitive forces just as during normal chewing, and also fatigue more often causes root fractures than static forces [24]. The accelerated fatigue testing method we used was based on the protocol of Magne et al. [20, 21]. This method represents an attempt to strike a balance between the load-to-fracture test and the more sophisticated and time-consuming fatigue tests [25]. Similar to other in vitro mechanical studies, the $135^{\circ}$ loading angle was used to simulate load applied to the lingual area of an upper anterior tooth $[7,11,26]$.

While Group 2 did not differ in survival from the intact teeth (control group), all other groups showed significantly lower survival rates compared to intact teeth. This could 
be due to the unique properties and high amount of fibers in the flowable SFRC. The efficacy of fiber reinforcement depends on several factors, including the resins used, the length, orientation and the position of the fibers, the aspect ratio, the adhesion of the fibers to the polymer matrix, and the impregnation of the fibers into the resin [27]. The aspect ratio refers to the length compared to the diameter of the fiber $(1 / \mathrm{d})$. The aspect ratio is of major importance in the case of advanced fiber-reinforced materials as it affects the tensile strength, flexural modulus, and the reinforcing efficiency of the material [28]. While packable SFRC utilizes millimeter-long fibers, the fibers in the flowable SFRC are micrometer-long. Although the fibers in the flowable material are smaller, the fiber length is equal to or greater than the critical fiber length and the aspect ratio is within the range of 30-94 [15], thus providing reinforcement to the materials and possibly to the adhered dental tissues. It is not less noteworthy that flowable SFRC contains $25 \mathrm{wt} \%$ of fibers, while packable SFRC only contains $9 \mathrm{wt} \%$. This could be a potential explanation of our findings regarding the survival in Group 2.

In the Bioblock technique, packable SFRC is directly and closely adapted to the root canal wall, eliminating the drawbacks of the usage of luting cement or the biomechanically incorrect positioning of the FRC post, thus potentially diminishing all the damaging tensile stresses produced when the restoration is loaded [14]. Although the Bioblock technique (Group 1) did not differ in survival from the flowable SFRC group (Group 2), it showed a significantly lower survival rate than intact teeth. In our previous study on restoring root canal treated premolars with MOD cavities, the packable SFRC inside the root canal produced significantly higher fracture resistance compared to teeth restored with FRC post [14]. This apparently contradicts the findings of Forster et al., where packable SFRC inside the root canal did not differ significantly from the teeth restored with a conventional FRC post in fracture resistance [13]. It must be added, though, that the study examined exclusively root canal treated premolars with class I cavities and used a static load-to-fracture setup. In the present study, there was no difference between the groups restored with different types of posts (conventional or individually-made) in terms of survival. This seems to complement our previous findings regarding fracture resistance (a proxy of survival) [29]. The reinforcing effect of FRC posts in immature teeth is a matter of ongoing debate. According to Jamshidi et al., and Linsuwanont et al. FRC posts cannot reinforce an immature tooth as the fracture resistance after post insertion is significantly lower compared to intact teeth $[8,11]$. In contrast, in the study of Schmoldt et al. immature teeth restored with FRC post showed higher fracture resistance compared to intact teeth [7]. It must be noted that all these studies used static load-to-fracture testing. In our study, FRC posts failed to reinforce immature interior teeth. As shown by Ambica et al. cyclic loading reduces the fracture strength of root canal treated teeth restored with FRC post [30]. Theoretically, long continuous fibers in the form of posts should be more suitable for the anterior region, as the forces in this region act nearly perpendicular to the fibers. Still, we found that long continuous fibers could not efficiently reinforce or increase the survival of immature teeth. Furthermore, they did not outperform the dual-cure core build-up material applied by itself either (Group 5). The reason behind the inferior performance of FRC posts in this situation compared to intact teeth is presumably that they fit inadequately in the critical cervical part of the already weakened teeth. If the post does not fit well, especially at the cervical level, the resin cement layer can be excessively thick, and bubbles are likely to form in it, which can lead to de-bonding $[31,32]$. This seems to be the case when looking at our current and previous results [14] on microgap formation in case of conventional FRC post, as this group (Group 4) produced a high amount of microgaps at the interface. As pointed out by Vallittu et al., the amount of fibers should be maximized in the critical cervical region and the amount of luting cement should be minimized [33]. Though the posts used in our groups (Groups 3 and 4) were large, they failed to reinforce the already weakened immature teeth.

Looking at the sectioned samples, the survival does not seem to be directly correlated with marginal microgap formation at the interface inside the canal. The packable version of SFRC (Group 1) had remarkably good adaptation to the canal walls of all the tested materials, which was in accordance with our previous findings regarding premolars [14]. This finding is also in accordance with the concept of the monoblock theory, which states that it is always beneficial to reduce the number of interphases as they do not only concentrate but also increase the amount of stress inside a restoration [34]. While the adaptation of the packable SFRC to the wall was ideal, gaps and voids were frequently found within the material itself. This has also been noticed by clinicians when using packable SFRC in a bulk manner. The authors' opinion is that these bubbles could be partly a sign of internal stress relief as shrinkage of the material is mandatory during its setting, and if shrinkage does not occur at the interface, it might cause internal voids inside the bulk material. In addition, some of these microgaps could be due to poor compression of the material inside the narrow space, or entrapment of air when applying the material into the canal. Interestingly, in the flowable SFRC group (Group 2 ), the only group that did not differ from intact teeth in terms of survival, the adaptation and subsequent microgap formation was less ideal. This could be caused by the flowable consistency of the material resulting in $3.3 \%$ volumetric shrinkage, which is higher than in the case of the packable version of SFRC. 
The microhardness values show that even the SFRC materials could be cured properly, nearly reaching the microhardness levels of dual-cure material. This is in accordance with our previous findings showing that SFRC can be lightcured inside the canal [14]. As shown by Garoushi et al. [35] and Lempel et al. [36], SFRC can be light-cured to a depth of $4-5 \mathrm{~mm}$. This is caused by both the translucency of the material and the fact that the randomly oriented fibers within it may conduct and scatter the light over longer distances [37]. Interestingly, in this study the microhardness values were higher compared to our previous findings in premolar teeth [14]. This might be traced back to the wider root canals in immature teeth, which may make it possible for more light to pass apically along the canal. Furthermore, due to the wider root canal a wider FRC post was used for light transmission during the Bioblock technique (Groups 1 and 2), which could theoretically transfer a greater amount of energy apically compared to smaller sized FRC posts.

\section{Conclusions}

The restoration of immature interior teeth with the use of flowable SFRC as post-core material displayed promising performance in a matter of fatigue resistance and survival.

Funding This study belongs to and was supported by the research activity of BioCity Turku Biomaterials Research Program in Turku, Finland and by the UNKP-19-2 New National Excellence Program of The Ministry of Human Capacities, Hungary.

\section{Compliance with ethical standards}

Conflict of interest Author Márk Fráter declares that he has no conflict of interest. Author Tekla Sáry declares that he has no conflict of interest. Author Viktória Néma Author declares that he has no conflict of interest. Author Gábor Braunitzer declares that he has no conflict of interest. Author Pekka Vallittu declares that he consults Stick TechMember of GC in training and RD. Lippo Lassila declares that he has no conflict of interest. Author Sufyan Garoushi declares that he has no conflict of interest.

Ethical approval This article does not contain any studies with human participants or animals performed by any of the authors.

\section{References}

1. O'Mullane DM. Injured permanent incisor teeth: an epidemiological study. J Irish Dent Assoc. 1972;18:160-73.

2. Ravn JJ. Dental injuries in copenhagen school children, school years 1967-1972. Community Dent Oral Epidemiol. 1974;2:231-45.

3. Wilkinson KL, Beeson TJ, Kirkpatrick TC. Fracture resistance of simulated immature teeth filled with resilon, gutta-percha, or composite. J Endod. 2007;33(4):480-3.
4. Andreasen FM, Andreasen JO, Bayer T. Prognosis of root-fractured permanent incisors: prediction of healing modalities. Endod Dent Traumatol. 1989;5:11-22.

5. Andreasen JO, Andreasen FM, Bakland LK, Flores MT. Traumatic dental injuries, a manual 2nd ed, vol. 9. Oxford: Blackwell Munksgaard; 2003. p. 58-60.

6. Witherspoon DE, Small JC, Regan JD, Nunn M. Retrospective analysis of open apex teeth obturated with mineral trioxide aggregate. J Endod. 2008;34:1171-6.

7. Schmoldt SJ, Kirkpatrick TC, Rutledge RE, Yaccino JM. Reinforcement of simulated immature roots restored with composite resin, mineral trioxide aggregate, gutta-percha, or a fiber post after thermocycling. J Endod. 2011;37(10):1390-3.

8. Jamshidi D, Homayouni H, Moradi Majd N, Shahabi S, Arvin A, Ranjbar Omidi B. Impact and fracture strength of simulated immature teeth treated with mineral trioxide aggregate apical plug and fiber post versus revascularization. J Endod. 2018;44(12):1878-82.

9. Cvek M. Prognosis of luxated non-vital maxillary incisors treated with calcium hydroxide and filled with gutta-percha. A retrospective clinical study. Endod Dent Traumatol. 1992;8(2):45-55.

10. Elnaghy A, Elsaka S. Fracture resistance of simulated immature roots using Biodentine and fiber post compared with different canal-filling materials under aging conditions. Clin Oral Investig. 2019. https://doi.org/10.1007/s00784-019-03014-8 [Epub ahead of print]

11. Linsuwanont P, Kulvitit S, Santiwong B. Reinforcement of simulated immature permanent teeth after mineral trioxide aggregate apexification. J Endod. 2018;44(1):163-7.

12. Garoushi S, Vallittu PK, Lassila LV. Continuous and short fiber reinforced composite in root post-core system of severely damaged incisors. Open Dent J. 2009;18(3):36-41.

13. Forster A, Sáry T, Braunitzer G, Fráter M. In vitro fracture resistance of endodontically treated premolar teeth restored with a direct layered fiber-reinforced composite post and core. J Adhes Sci Technol. 2016;31:1454-66. https://doi.org/10.1080/01694 243.2016.1259758.

14. Fráter M, Lassila L, Braunitzer G, Vallittu PK, Garoushi S. Fracture resistance and marginal gap formation of post-core restorations: influence of different fiber-reinforced composites. Clin Oral Investig. 2019. https://doi.org/10.1007/s00784-019-02902-3 [Epub ahead of print].

15. Lassila L, Keulemans F, Säilynoja E, Vallittu PK, Garoushi S. Mechanical properties and fracture behavior of flowable fiber reinforced composite restorations. Dent Mater. 2018;34(4):598-606.

16. Garoushi S, Vallittu P, Lassila L. Mechanical properties and radiopacity of flowable fiber-reinforced composite. Dent Mater J. 2019;38(2):196-202.

17. Lassila L, Säilynoja E, Prinssi R, Vallittu P, Garoushi S. Characterization of a new fiber-reinforced flowable composite. Odontology. 2019;107(3):342-52.

18. Scotti N, Coero Borga FA, Alovisi M, Rota R, Pasqualini D, Berutti E. Is fracture resistance of endodontically treated mandibular molars restored with indirect onlay composite restorations influenced by fibre post insertion? J Dent. 2012;40(10):814-20.

19. Le Bell AM, Tanner J, Lassila LV, Kangasniemi I, Vallittu P. Bonding of composite resin luting cement to fiber-reinforced composite root canal posts. J Adhes Dent. 2004;6(4):319-25.

20. Magne P, Lazari PC, Carvalho MA, Johnson T, Del Bel Cury AA. Ferrule-effect dominates over use of a fiber post when restoring endodontically treated incisors: an in vitro study. Oper Dent. 2017;42(4):396-406.

21. Lazari PC, de Carvalho MA, Del Bel Cury AA, Magne P. Survival of extensively damaged endodontically treated incisors restored with different types of posts-and-core foundation restoration material. J Prosthet Dent. 2018;119(5):769-76. 
22. Fonseca RB, Haiter-Neto F, Carlo HL, Soares CJ, Sinhoreti MA, Puppin-Rontani RM, Correr-Sobrinho L. Radiodensity and hardness of enamel and dentin of human and bovine teeth, varying bovine teeth age. Arch Oral Biol. 2008;53(11):1023-9.

23. Reis AF, Giannini M, Kavaguchi A, Soares CJ, Line SR. Comparison of microtensile bond strength to enamel and dentin of human, bovine, and porcine teeth. J Adhes Dent. 2004;6(2):117-21.

24. Le Bell-Rönnlöf AM, Lassila LV, Kangasniemi I, Vallittu PK. Load-bearing capacity of human incisor restored with various fiber-reinforced composite posts. Dent Mater. 2011;27(6):e107-15.

25. Magne P, Carvalho AO, Bruzi G, Anderson RE, Maia HP, Giannini $\mathrm{M}$. Influence of no-ferrule and no-post buildup design on the fatigue resistance of endodontically treated molars restored with resin nanoceramic CAD/CAM crowns. Oper Dent. 2014;39:595-602.

26. Tanalp J, Dikbas I, Malkondu O, Ersev H, Güngör T, Bayırlı G. Comparison of the fracture resistance of simulated immature permanent teeth using various canal filling materials and fiber posts. Dent Traumatol. 2012;28(6):457-64.

27. Garoushi S, Gargoum A, Vallittu PK, Lassila L. Short fiber-reinforced composite restorations: a review of the current literature. J Investig Clin Dent. 2018;9(3):e12330.

28. Bijelic-Donova J, Garoushi S, Lassila LV, Keulemans F, Vallittu PK. Mechanical and structural characterization of discontinuous fiber-reinforced dental resin composite. J Dent. 2016;52:70-8.

29. Fráter M, Forster A, Jantyik Á, Braunitzer G, Nagy K, Grandini $\mathrm{S}$. In vitro fracture resistance of premolar teeth restored with fibre-reinforced composite posts using a single or a multi-post technique. Aust Endod J. 2017;43(1):16-22.

30. Ambica K, Mahendran K, Talwar S, Verma M, Padmini G, Periasamy R. Comparative evaluation of fracture resistance under static and fatigue loading of endodontically treated teeth restored with carbon fiber posts, glass fiber posts, and an experimental dentin post system: an in vitro study. J Endod. 2013;39(1):96-100.

31. Faria-e-Silva AL, Pedrosa-Filho Cde F, Menezes Mde S, Silveira DM, Martins LR. Effect of relining on fiber post retention to root canal. J Appl Oral Sci. 2009;17(6):600-4.

32. Grandini S, Goracci C, Monticelli F, Borracchini A, Ferrari M. SEM evaluation of the cement layer thickness after luting two different posts. J Adhes Dent. 2005;7(3):235-40.

33. Vallittu PK. Are we misusing fiber posts? Guest editorial. Dent Mater. 2016;32(2):125-6.

34. Belli S, Eraslan O, Eskitascioglu G, Karbhari V. Monoblocks in root canals: a finite elemental stress analysis study. Int Endod J. 2011;44(9):817-26.

35. Garoushi S, Säilynoja E, Vallittu PK, Lassila L. Physical properties and depth of cure of a new short fiber reinforced composite. Dent Mater. 2013;29(8):835-41.

36. Lempel E, Őri Z, Szalma J, Lovász BV, Kiss A, Tóth Á, KunságiMáté $S$. Effect of exposure time and pre-heating on the conversion degree of conventional, bulk-fill, fiber reinforced and polyacidmodified resin composites. Dent Mater. 2019;35(2):217-28.

37. Li X, Pongprueksa P, Van Meerbeek B, De Munck J. Curing profile of bulk-fill resin-based composites. J Dent. 2015;43(6):664-72.

Publisher's Note Springer Nature remains neutral with regard to jurisdictional claims in published maps and institutional affiliations. 